\title{
Intelligenter als die Konkurrenz
}

Der Duden beschreibt Intelligenz als die „Fähigkeit (des Menschen), abstrakt und vernünftig zu denken, um daraus zweckvolles Handeln abzuleiten“. Können Marketing und Vertrieb an sich überhaupt intelligent sein? Oder haben wir mit dem Titel dieser Ausgabe, „Marketing- und Vertriebsintelligenz“, eine Art Buzzword kreiert?

Eine rhetorische Frage, denn am Ende sind es - wie überall in Organisationen - auch im Marketing und im Vertrieb Menschen, die planen, entscheiden und umsetzen. In dieser Ausgabe haben wir interessante Beiträge zusammengestellt, die Führungskräfte und Mitarbeiter in Marketing und Vertrieb dabei unterstützen, intelligent zu arbeiten. Das Thema ist jedoch kein Selbstzweck: Es geht immer darum, aus dem Blickwinkel der Kunden besser zu sein als die Wettbewerber. Dazu benötigt der Mensch Mechanismen und Rahmenbedingungen im Unternehmen, wie z. B. die IT-Infrastruktur. Das Interview mit Andreas Stuker, COO der SAP-Schweiz, macht die Breite des Themas deutlich.

Aus strategischer Sicht bieten wir Ihnen mit der „Value-toValue"-Segmentierung" einen innovativen Ansatz zur Operationalisierung der zwei Wertperspektiven des Customer-Value-Managements. Weitergehend stellen wir eine neue Leitzielgruppe unserer globalen und vernetzten Welt vor, die getrieben von Individualität und Autonomie eine neue kosmopolitische Elite entstehen lässt: die Digitale Avantgarde.

Marketing- und Vertriebsintelligenz benötigt verlässliche Informationen über Kunden, um adäquat und marktgerecht reagieren zu können. Diese kommen nicht nur aus der Marktforschung, sondern auch direkt aus Vertrieb, Key Account Management oder sogar den sozialen Medien. So thematisieren wir zum einen, wie Key Account Manager als Schlüssel zur Gewinnung strategisch relevanter Informationen genutzt werden können. Zum anderen zeigen wir, wie die Auswertung von Kundeninteraktionen in sozialen Medien im Sinne eines "Social Screenings“ Mehrwert generiert. Auch die Themen „Big Data“ und „E-Business“ fehlen im Kontext dieser Ausgabe nicht. Wir zeigen insbesondere auf, wie Big Data bei KMUs umgesetzt werden kann und welche Faktoren aus Kundensicht beim Online-Vertrieb von Automobilen zu berücksichtigen sind.

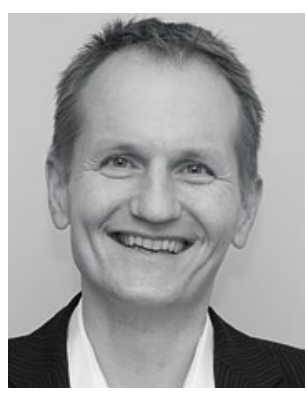

Prof. Dr. Sven Reinecke

Geschäftsführender Direktor

des Instituts für Marketing an der

Universität St. Gallen (HSG)

sven.reinecke@unisg.ch

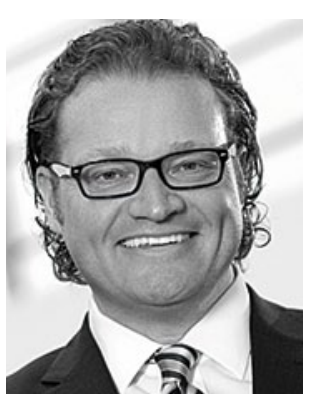

Prof. Dr. Dirk Zupancic

Präsident der German Graduate School of Management and Law (GGS) in Heilbronn sowie Professor für Industriegütermarketing und Vertrieb dirk.zupancic@ggs.de

Die Rubrik Spektrum rundet die Ausgabe mit einem Fallbeispiel zum Veränderungsmanagement bei Bayer CropScience und einem Beitrag zur Markenstärke ab.

Wir wünschen Ihnen eine spannende Lektüre und viele Impulse zur Steigerung Ihrer eigenen Marketing- und Vertriebsintelligenz. Wir freuen uns auch über Rückmeldungen zu dieser Ausgabe und Anregungen zu weiteren Themen.

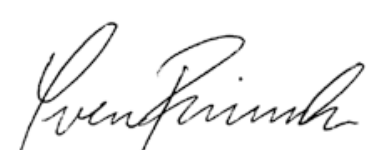

Sven Reinecke

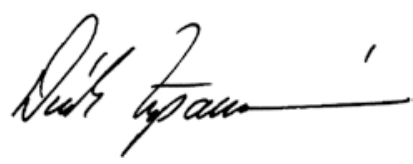

Dirk Zupancic 